\title{
Tailoring the Ti surface via electropolishing nanopatterning as a route to obtain highly ordered $\mathrm{TiO}_{2}$ nanotubes
}

\author{
A Apolinário, ${ }^{1} \mathrm{C}$ T Sousa,,${ }^{1} \mathrm{~J}$ Ventura, ${ }^{1} \mathrm{~L}$ Andrade,${ }^{2} \mathrm{~A} \mathrm{M}$ Mendes ${ }^{2}$ and J P Araújo ${ }^{1 *}$ \\ ${ }^{1}$ IFIMUP and IN- Institute of Nanoscience and Nanotechnology, Departamento de Física e \\ Astronomia, Faculdade de Ciências da Universidade do Porto, Rua do Campo Alegre, 678, 4169- \\ 007 Porto, Portugal. \\ ${ }^{2}$ LEPAE- Dep. Engenharia Química-Faculdade de Engenharia, R. Dr. Roberto Frias, 4200-465 \\ Porto, Portugal \\ *E-mail: jearaujo@fc.up.pt
}

\begin{abstract}
Highly-ordered $\mathrm{TiO}_{2}$ nanotubes (NTs) were synthesized by the electrochemical anodization of $\mathrm{Ti}$ foils subjected to electropolishing (EP) pre-treatment. We found that the $\mathrm{Ti}$ surface roughness plays an important role in the onset of pore nucleation in enhancing the local focusing effect of the electrical field. Additionally, EP induces the formation of dimple structures on the metal surface, which can work as a pre-pattern prior to the anodization. These shallow ripples lead to a preferential ordered pore nucleation, offering an organization improvement of the anodic oxide NTs. We found that, depending on the EP applied potential, the roughness and the spacial period of the ripple-like structures varies from 8 to $2 \mathrm{~nm}$ and from 122 to $30 \mathrm{~nm}$, respectively. Such tuning allowed us to focus on the influence of the initial Ti pre-surface topography features on the NTs length, organization and hexagonal arrangement quality, NTs diameter and density. Our results show that an EP under $10 \mathrm{~V}$ is the most suitable to obtain a small Ti surface roughness, the largest NT length ( $40 \%$ enhancement) and the effective improvement of the ordered hexagonal NTs arrays over larger areas. Furthermore, the NTs dimensions (pore diameters and density) were found to also depend on the initial Ti surface topography. The use of optimized EP allows to obtain highly hexagonal self-ordered samples at a reduced time and cost.
\end{abstract}

\section{Introduction}

The electrochemical anodization of Ti to obtain highly-ordered nanotube (NT) arrays of $\mathrm{TiO}_{2}$ continues to gain an increasing importance for a large number of applications, such as photoelectrochemical cells for $\mathrm{H}_{2}$ production (water splitting) [1,2] and particularly for dye-sensitized solar cells (DSCs) [2]. $\mathrm{TiO}_{2} \mathrm{NTs}$ have received great attention due to their one dimensional structure which provides a direct and efficient path for electrons [1].

$\mathrm{TiO}_{2} \mathrm{NTs}$ can be obtained by the electrochemical anodization of $\mathrm{Ti}$ in fluoride-based electrolytes. The addition of ethylene glycol was later found to lead to longer (hundreds of microns) and more ordered arrays of $\mathrm{TiO}_{2}$ NTs [3-9]. Differently from the well-known case of Al anodization, the Ti anodization presents a non-steady state, due to the out of equilibrium dissolution and oxidation processes on the NT bottom and to the additional chemical dissolution process at the NTs top [5,10-14]. This unbalanced anodization leads to a maximum $\mathrm{TiO}_{2} \mathrm{NTs}$ length, after a given anodizing time. Besides the length, other NT parameters are influenced by factors such as the electrolyte type (e.g. electrolyte concentration and $\mathrm{pH})$, anodization temperature and applied potential [3-10,15-18].

Aiming a degree of order comparable to that of anodic alumina oxide (AAO) at $10 \%$ porosity [19], intense research has been performed on the organization and final ordering of $\mathrm{TiO}_{2} \mathrm{NTs}$ in a closed- 
packed hexagonal array [20-24]. The organization of $\mathrm{TiO}_{2} \mathrm{NTs}$ is mainly influenced by the Ti foil purity, the anodization voltage [21] and the local initial surface morphology of the Ti substrate [22-25]. Regular or periodic dimples in a valve metal surface can lead to an improvement of the organization degree of NT arrays, inhibiting their random nucleation $[25,26]$. In this context, the two-step anodization process was found to be mandatory to improve organization in AAO and has been widely used to improve the structural order of $\mathrm{TiO}_{2} \mathrm{NT}$ arrays [20,21,22,27]. On the other hand, an electropolishing (EP) pretreatment of the metal foil prior to the anodization was shown to be highly suitable to induce a high degree of organization in AAO [27]. In the case of $\mathrm{TiO}_{2} \mathrm{NT}$ arrays, several pre-treatments (such as EP, mechanical polishing or chemical etching) were also studied targeting smoother Ti surfaces. This ensures that a highly homogeneous electric field distribution is obtained over the Ti surface during the initial anodization stages, a crucial condition to reach highly ordered NTs arrays [22,23,24,28]. In particular, EP pre-treatments were shown to decrease the Ti foil surface roughness, resulting in more uniform NTs length and higher NTs density [22,23,24,28].

Electropolishing is a well-known technique [29] for metal removal at the micrometer level [30-34] and to obtain smooth and highly reflective surfaces. While (mostly) at the industrial level the process is best known for the surface bright polish, at the research level other important benefits, often overlooked, arise. Besides usual advantages, like microfinishing improvement or size control, the EP surface "waviness"

offers great promise to improve the organization of anodic oxide nanopores or nanotubes. A new overriding application of EP arises to create nanopatterns in the surface of aluminum, titanium, zirconium, tantalum and tungsten $[32,33]$, working as a pre-pattern prior to the anodization and ultimately leading to highly ordered oxide nanostructures. In fact, the EP of Al foils was shown to be a simple and efficient method to induce a high degree of organization in AAO templates, since the produced shallow ripple-like structures favor a preferential ordered pore nucleation [27,33,34,36-40]. In Al, the EP process has been widely studied and optimized and EP parameters, such as temperature, time, stirring, distance to the electrode and electrolyte type, were found to play an important role in the process $[35,36]$. Nevertheless, a parameter of crucial important is the EP applied voltage since its variation can result in features like stripes or hexagonally ordered dimples (from 50 to $150 \mathrm{~nm}$ ) [33-36,40]. However, the nanopatterning of a Ti foil by varying the EP potential has been overlooked so far. EP of Ti is usually performed using sulphuric acid-based electrolytes, which results in smooth surfaces [31], although other $\mathrm{EP}$ conditions (e.g. $\mathrm{H}_{2} \mathrm{SO}_{4} / \mathrm{HF}$ electrolyte under an applied voltage of $15 \mathrm{~V}$ ) lead to organized dimplepattern structure at the Ti surface [33].

In this work the growth of $\mathrm{TiO}_{2}$ NTs is optimized by tuning the Ti foil surface roughness using different EP conditions, in particular adjusting the EP applied potential $\left(V_{\mathrm{EP}}\right)$. An integrated approach, based on previously optimized anodization parameters [3-18,20-26] and directly focused on the effect of the Ti surface roughness and topographic features on the length and organization of the obtained NT arrays was here followed. By varying $V_{\mathrm{EP}}$, and comparing with an as-rolled (AR) Ti foil, our results show the critical role played by the Ti surface topography and roughness prior to the anodization on the maximum NT length and their organization in a regular hexagonal pattern. By tuning $V_{\mathrm{EP}}$, adequate EP conditions were found that enables highly ordered hexagonal closed-packed arrays of $\mathrm{TiO}_{2} \mathrm{NTs}_{\mathrm{s}}$ with lengths above $100 \mu \mathrm{m}$.

\section{Materials and methods}

Ti foils $(0.127 \mathrm{~mm}$ thick, $99.99+\%$ purity from AlfaAesar $)$ pieces $\left(1 \mathrm{~cm}^{2}\right)$ were ultrasonically cleaned in ethanol and deionized water for 10 minutes. Prior to the anodization, the pieces of the as-rolled Ti foil were pre-treated by an EP in a $\mathrm{H}_{2} \mathrm{SO}_{4} / \mathrm{HF}(8: 3)$ solution, under applied potentials $\left(V_{\mathrm{EP}}\right)$ between the Ti foil and an inert $\mathrm{Pt}$ mesh (at a distance of $2.5 \mathrm{~cm}$ ) for $4 \mathrm{~min}$. Several samples were fabricated with different $V_{\mathrm{EP}}$ of $5,10,15,18$ and $20 \mathrm{~V}$; the solution was mechanically stirred $(300 \mathrm{rpm})$ and kept at room temperature $[33,41]$. The Ti surfaces of all electropolished samples, as well as an as-rolled Ti sample, were analyzed using a Nanoscope Multimode Atomic Force Microscope from Veeco Instruments. 
Subsequently, the samples were electrochemically anodized in a home-made anodization cell (twoelectrodes), where the Ti foil acted as the anode and an inert Pt mesh was used as cathode. The time evolution of the current density [j(t)] was monitored during the anodization using a Keithley 2004 Sourcemeter remotely controlled by a LabView application. The electrochemical anodization was carried out for all samples at a constant potential of $60 \mathrm{~V}$ for $17 \mathrm{~h}$, in a freshly prepared ethylene glycol solution containing $\mathrm{NH}_{4} \mathrm{~F}(0.3 \mathrm{wt} \%)$ and $\mathrm{H}_{2} \mathrm{O}(2 \mathrm{wt} \%)$ at room temperature with mechanical stirring [11]. The NTs morphology was evaluated by a FEI Quanta 400FEG Field Emission Scanning Electron Microscopy (SEM) using surface top and bottom views and cross-sections for the NTs length calculation. The bottom views were obtained by natural peeling-off originating free-standing and non-curling membranes. Using SEM images, a statistical analysis (with the ImageJ image processing software) [42] was performed, enabling an evaluation of the organization pattern and NT structure dimensions (diameters and density).

\section{Results and discussion}

\subsection{AFM imaging analyses: surface topography and roughness characterization}

The surface topography of the Ti samples with EP pre-treatments at different $V_{\mathrm{EP}}$ and one as-rolled was investigated by AFM imaging. 1 x $1 \mu \mathrm{m}^{2}$ topographic images of Ti surfaces were obtained for each sample and then analyzed in terms of surface roughness $(R q)$, defined as the root-mean-square value of the images pixel height. Figure 1 shows illustrative AFM images of the Ti surfaces of each sample, as well as the $R q$ values extracted from the measured topography. For the unpolished sample (AR) we observe an irregular surface with a roughness of $8.3 \mathrm{~nm}$, that is slightly increased upon a $5 \mathrm{~V}$ EP [Figs. 1(a) and (b)]. Nonetheless, the latter shows broad random topographic features (hills), whereas the former shows steeper hills. In contrast, a significantly smoother surface was obtained in other EP samples, particularly the $10 \mathrm{~V}$ sample with a $R q$ of $2.4 \mathrm{~nm}$ [Fig. 1 (c)]. By increasing $V_{\mathrm{EP}}$, the Ti foil surface became slightly rougher: $R q=3.4,4.7$ and $3.1 \mathrm{~nm}$ measured for samples 15,18 and $20 \mathrm{~V}$, respectively. Additionally, the Ti surface after EP revealed a periodic dimple-pattern structure whenever $V_{\mathrm{EP}}$ was no smaller than $10 \mathrm{~V}$. This shallow ripple-like structure is an EP characteristic of metals $[33,41]$ and can be used as a pre-pattern prior to the anodization to obtain highly ordered oxide nanostructures, as profusely shown in the case of $\mathrm{Al}$ [27,33-36,40]. In fact, studies in Al foils demonstrated that, by combining adequate EP voltage and time, dimple-patterns from striped to hexagonal arrangements can be obtained $[27,33-36,40]$. However, EP of Ti is usually performed in sulphuric acid-based electrolytes that leads to smooth and plane surfaces [31]. Nonetheless, EP with HF-electrolytes can also result in organized dimple patterns under certain conditions (time, temperature, voltage) $[33,41]$.

Our results clearly demonstrate that the EP voltage also determines the ripple-like structures of the Ti surface, particularly the inter-ripple spacial period $(S P)$. Figure 1(e) shows AFM images profiles of each $\mathrm{V}_{\mathrm{EP}}$ sample, giving the mean inter-ripple spacial periods: $S P_{5 \mathrm{~V}}=122 \mathrm{~nm}, S P_{10 \mathrm{~V}}=97 \mathrm{~nm}, S P_{15 \mathrm{~V}}=194 \mathrm{~nm}$, $S P_{18 \mathrm{~V}}=30 \mathrm{~nm}$ and $S P_{20 \mathrm{~V}}=73 \mathrm{~nm}$. The variation of the Ti surface waviness with $V_{\mathrm{EP}}$ allows its adequate tuning for particular purposes; in our case we will demonstrate that it makes possible the creation of selforganized hexagonal and long $\mathrm{TiO}_{2}$ NTs by a single electrochemical anodization. Ti pre-pattern can be the vehicle to achieve in an easy way ordered hexagonally arranged NTs and to enhance the NTs growth rate, avoiding the later chemical effects that limit the NTs growth.

\subsection{Anodization curves}

1.2.1.Typical growth mechanism. The evaluation of the mechanisms that lead to the formation and growth of the NTs can be studied using current density $(j)$ curves [41]. For the samples AR, $5 \mathrm{~V}$ and $10 \mathrm{~V}$, these curves present the typical behaviour for Ti anodization and the successful formation of $\mathrm{TiO}_{2}$ nanotubes (Fig. 2) $[3,4,11,20,23]$. After applying the anodization potential $(60 \mathrm{~V})$, a continuous $\mathrm{TiO}_{2}$ layer is rapidly formed increasing the resistance (rapid $j$ decrease). The following slight $j$-decrease marks the onset of NT nucleation, likely on the surface valley-type irregularities where the higher electric field enhances oxide dissolution and hole formation (dissolution promoted by $\mathrm{F}^{-}$ions in favourable spots of the $\mathrm{TiO}_{2}$ surface)[41]. Consequently, the $\mathrm{TiO}_{2}$ layer thickness starts to decrease, while the pores (tubes) formation 
accelerates. This is evidenced by the increase of $j$ until a maximum is reached $\left(j_{\max }\right)$, forming a barrier layer at the NTs bottom. Afterwards, the emerging NTs will adjust and compete with each other in a selforganization process.

As the anodization proceeds, the formed NTs grow at a constant rate and the barrier layer thickness $\left(\delta_{b}\right)$ would remain constant if Ti oxidation balanced oxide dissolution $[3,4,8,10]$. However, at this stage, the oxidation/dissolution processes are not in equilibrium, being the $\mathrm{TiO}_{2}$ dissolution lower than its formation, resulting in a slow decay of $j(t)$ during the anodization. This is associated with the progressive depletion of F- ions (which are crucial to the electrolyte/oxide interface dissolution process) at the NT bottoms during the anodization. Given the high viscosity of the electrolyte and increasing length of the NTs (diffusion limited process) such ions are difficult to replace by new ones at the NT bottom. As a result, the oxidation process is faster than dissolution ( $j$ slowly decrease with time), leading to a progressive increase of the oxide $\delta_{\mathrm{b}}$ at the NTs' bottom. Thus, the ionic migration path along the oxide barrier [8] significantly extends, inhibiting the transport of $\mathrm{F}^{-}, \mathrm{Ti}^{4+}$ and $\mathrm{O}^{2-}$ ions across $\delta_{\mathrm{b}}\left(\mathrm{Ti}^{4+}\right.$ and $\mathrm{O}^{2-}$ for oxidation, $\mathrm{F}^{-}$for dissolution) which subsequently limits further NT growth.

1.2.2.Dependence of the anodization curves on different electropolishing voltages. According to the $j(t)$ behaviours shown in Fig. 2, we can divide the produced samples into two groups: the AR sample and samples with lower $V_{\mathrm{EP}}(5 \mathrm{~V}$ and $10 \mathrm{~V})$ present lower $j$-values and the initial transient regime typical of valve metals anodization; and samples with $V_{\mathrm{EP}} \geq 15 \mathrm{~V}$ that have higher $j$-values and an absence of initial $j$-transients.

For the first group of samples the $j(\mathrm{t})$ curves show the typical behaviour [3,4,11,20,23] of mildanodizations (MA; anodizations under low potentials, $<60 \mathrm{~V})[20,43,44]$. Within this group, samples with higher $R q(5 \mathrm{~V}, 9.13 \mathrm{~nm}$ and $\mathrm{AR}, 8.3 \mathrm{~nm})$, have lower $j$-values indicating lower ionic current during the anodization. Thus, high $R q$ hinders $\mathrm{F}^{-}$to reach the surface and causes an inhomogeneous electric field over the samples area, which is a crucial parameter for a proper oxide dissolution. At this point, the oxidation/dissolution processes are not in equilibrium (non-steady-state anodization), being the $\mathrm{TiO}_{2}$ dissolution lower than its formation, which limits further NT growth. The AR and $5 \mathrm{~V}$ samples present similar $R q$ (although $R q$ of the $5 \mathrm{~V}$ sample is slightly higher since HF works as a chemical etcher of the Ti surface) $[41,45]$ but different topographic features that cause the differences in the $j$ values and initial transient period. As showed in the AFM results, the $5 \mathrm{~V}$ sample presents higher and broader random topographic features than the AR sample that in turn shows much steeper hills. This allows the formation of a thicker initial oxide layer (lower $j$ ) and longer transient period (nucleation, oxide dissolution, tubeformation stages). On the other hand, the AR sample presents steep nucleation spots that allow a faster nucleation and oxide dissolution and thus a more rapid formation of the NTs.

The $10 \mathrm{~V}$ sample shows the lowest surface $R q(2.4 \mathrm{~nm})$, a reduction of $70 \%$ when compared to that of AR. Its anodization curve reveals a more stable $j(t)$ which is fundamental for a longer balance of the oxidation/dissolution processes and indicates higher NTs growth rate and thus longer NTs. Besides, this sample reveals unique periodical rippled structures [Fig. 1(c)] that are a key characteristic for pore formation since they act as nucleation points (valleys) making the overall nucleation time short. This allows rapid oxide dissolution, leading to a pronounced $j_{\max }$ (inset of Fig. 2), a shoulder-like trend which is usually ascribed to an optimized hexagonal arrangement of the network. Thus, the ripples of the $10 \mathrm{~V}$ sample lead to earlier and more rapidly organized NT nucleation than the random valleys of the AR and 5 V samples, explaining the shorter nucleation and dissolution stages.

For the second group of samples $(15,18$ and $20 \mathrm{~V})$, the $j(t)$ curves show a different behaviour from those previously discussed due to topography effects. The main differences in $j(t)$ focuses on two critical aspects: i) a stronger monotonic decrease of $j(t)$ along the whole anodization period; (ii) the absence of the typical $j(t)$ transient related with the initial growth mechanisms of the $\mathrm{TiO}_{2} \mathrm{NTs}$.

Regarding the first point, the almost exponential $j(t)$ decay is typical of hard-anodization (HA) curves (anodizations under high potentials, $>60 \mathrm{~V}$ ) and indicates a predominance of oxidation over dissolution $[20,43,44]$. This HA behaviour can be ascribed to local burning defects on the Ti surface caused by the high EP potentials (15 - 20 V; Fig. 3)[46]. Under these conditions, the current will flow predominantly 
through the local-features resulting in a rapid growth of the oxide layer. The migration path of the ionic species along the barrier is then significantly extended and a non-steady state anodization occurs from the very beginning. Therefore, the stronger oxidation ionic current seen in the $j(t)$ curve decay of these samples encourages this type of HA behaviour.

It was recently reported [41] that a chemical etching pre-treatment also gives rise to anodization curves without the initial typical transient (NTs nucleation and growth). The severe chemical etching effects observed in that case damaged the surface, creating areas of different heights and NTs growth rate (higher at the valleys). The obtained macroscopic $j(t)$ curve is then the sum of the microscopic $j$ contributions from the different anodization zones. In this way, we may realize that the 15,18 and $20 \mathrm{~V}$ samples (presenting lower $R q$ and ripples) have a non-homogeneous surface topography over the total area. In particular, the lower $R q$ and ripples that promote rapid nucleation (as in the $10 \mathrm{~V}$ case) are masked by the anodization areas where the Ti surface was damaged by the high EP potentials.

\subsection{NTs length and organization}

1.3.1. Lenght. Figure 4 shows SEM cross-section and top view images of the NT templates for all samples after $17 \mathrm{~h}$ of anodization. The resulting template thicknesses $(L)$ are shown in Table 1 . The AR sample has a mean NT template thickness of $89 \mu \mathrm{m}$, while the $5 \mathrm{~V}$ sample has a slighter smaller thickness, $L=83$ $\mu \mathrm{m}$. On the other hand, for $V_{E P}=10$ and $15 \mathrm{~V}$ a rapid NT growth occurs, leading to a length increase of over $40 \%$ ( $L=125$ and $101 \mu \mathrm{m}$, respectively) when compared with the AR or $5 \mathrm{~V}$ samples. Nonetheless, by further increasing $V_{E P}, L$ decreases to 88.7 and $76.4 \mu \mathrm{m}$ for 18 and $20 \mathrm{~V}$, respectively.

Comparing $L$ with the $R q$ results, one can infer that the NTs growth and template thickness is directly correlated with the surface topography (Fig. 5). By decreasing $R q$, as for the 10 and $15 \mathrm{~V}$ samples, we achieved an enhancement of the growth rate (and $L$ ). In fact, the pre-anodization surface conditions with the lowest $R q$ of $2.4 \mathrm{~nm}$ and the presence of small nucleation sites are crucial to reach higher growth rates and thicker NTs $(125 \mu \mathrm{m})$. For samples with lower $R q$, we obtain a more homogeneous electric field distribution over the whole sample surface, causing a slower $\delta_{b}$ increase during the anodization. Such thinner $\delta_{b}$ then promotes an easier ionic electromigration $\left(\mathrm{F}^{-}\right.$and $\left.\mathrm{O}^{2-}\right)$ through the oxide layer, fundamental for the existence of balanced dissolution/oxidation processes and thus of steady state anodization. Also, smoother surface favours the mobility of the ions involved in the dominant anodization processes, promoting the availability of $\mathrm{F}^{-}$at the Ti surface (thus favouring dissolution). On the other hand, the additional chemical effects occurring later in the anodization (namely the $\mathrm{pH}$ decrease) are delayed since the anodization growth limit caused by the too thick $\delta_{b}$ is also belated.

As discussed before, further increasing $V_{E P}$ to $18 \mathrm{~V}$ and $20 \mathrm{~V}$, besides decreasing the $R q$ values and causing topographic ripple-like structures, also leads to areas with defects (burning) and cracks (Fig. 3). These defects relentlessly influence the anodization rate, decreasing the NTs growth rate and lowering the final $L$ compared to the AR sample. The anodizing current is, in this case, preferentially directed toward those defect and cracked areas, discouraging NTs growth on the rest of the surface sample. The current is thus not homogeneous in the whole sample resulting in a slower NT growth rate with final thinner and inhomogeneous [increase of standard deviation (SD) in the $20 \mathrm{~V}$ sample] thicknesses.

After $17 \mathrm{~h}$ of anodization the oxide nucleation layer of the $10 \mathrm{~V}$ sample remains almost intact, while those with larger $V_{\mathrm{EP}}$ have been partially dissolved (top view SEM images shown in Fig. 4) [41]. As the anodization proceeds, a local $\mathrm{pH}$ decrease occurs at the NTs bottom [11], with the electric field driving the $\mathrm{H}^{+}$ions towards the NTs top (V-shaped NTs) $[11,21,45]$, resulting in the oxide nucleation layer dissolution [41]. Consequently, the $\mathrm{F}^{-}$ions can again easily reach the bottom of the tubes, being more readily available to increase the NTs growth rate. This is clearly seen in the 15 and $20 \mathrm{~V} j(t)$ curves presented in the inset of Fig. 2, where a $j$-increase is observed at instants $820 \mathrm{~min}$ and $940 \mathrm{~min}$ respectively, indicating an upsurge in the NTs template thickness. Note that the smaller SP of the $18 \mathrm{~V}$ sample led to a rather homogeneous template. Nevertheless, top-view images revealed that the NTs top of this sample was also dissolved after $17 \mathrm{~h}$. 
Table 1. $\mathrm{TiO}_{2} \mathrm{NTs}$ template thickness results: mean $<L>$, maximum $L_{\max }$ length and $S D$.

\begin{tabular}{rrrrrrr} 
& $\mathrm{AR}$ & $5 \mathrm{~V}$ & $10 \mathrm{~V}$ & $15 \mathrm{~V}$ & $18 \mathrm{~V}$ & $20 \mathrm{~V}$ \\
\hline$<L>(\mu \mathrm{m})$ & 88.93 & 82.50 & 124.2 & 101.6 & 85.70 & 76.40 \\
$L_{\max }(\mu \mathrm{m})$ & 91.50 & 89.84 & 125.0 & 114.5 & 88.70 & 82.75 \\
$S D(\mu \mathrm{m})$ & 4.012 & 8.772 & 1.217 & 4.865 & 2.372 & 15.24 \\
\hline
\end{tabular}

1.3.2. NTs Organization, Diameter and Density. The degree of organization of the obtained NT arrays can be evaluated by the study of the NTs bottom. Since top analyses shows the first oxide nucleation pore distribution (that follows the initial non-optimized topography), the bottom analyses arises as the best method to evaluate organization $[20,23,41]$. The order-degree was evaluated as a function of the NTs hexagonal arrangement [ideal Hexagonal Closed-Packed Array (HCPA) structure] and their individual shape $[21,41]$. The NTs organization increases as they pack themselves in a closed-packed hexagonal arrangement, leading to tubes with a hexagonal section. The deviations from this shape due to Ti defects and grain boundaries result in pentagons, heptagons or circular shapes $[21,41]$.

Our results show that only the 10 and $18 \mathrm{~V}$ samples present both HCPA structure and NT of hexagonal shape (Fig. 6). Therefore, the samples with highest $R q$ (AR, $5 \mathrm{~V}$ ) and the 15 and $20 \mathrm{~V}$ samples present a smaller degree of organization since, although having some HCPA domains, the NTs shape is not a perfect hexagon.

The NTs organization is also related with the surface parameters. The combination between $R q$ and topographic periodic ripples, leads to an almost perfect organization. The $10 \mathrm{~V}$ sample presents the lowest $R q(2.4 \mathrm{~nm})$ with a SP of $97 \mathrm{~nm}$, while the $18 \mathrm{~V}$ sample has a $R q \sim 5 \mathrm{~nm}$ and $S P=30 \mathrm{~nm}$. These small topographic features lead to a more uniform electric field distribution causing a much easier NT nucleation and inducing an ideal NT arrays distribution from the beginning of the anodization. Thus, the smallest $S P$ could be considered as an important advantage in terms of NTs organization.

The $20 \mathrm{~V}$ sample, although having a similar $S P$ to that of the $10 \mathrm{~V}$ and a lower $R q$ than that of the $18 \mathrm{~V}$, shows worse organization (and smaller $L$ ) since these factors are shadowed by the EP potential-induced catastrophic defects. This leads to a non-efficient anodization due to the focusing of the electric field on these defects.

Based on the bottom NTs analysis, the NTs diameter $(D)$ and NTs density (number of NTs per unit area) were also quantified (Fig. 7). The average diameter was estimated from the size-histogram, which was then fitted to a log-normal distribution (the inset of Fig. 7 presents an example for the $10 \mathrm{~V}$ sample). The NTs density was calculated by counting the NTs in the same single domain area where $D$ was estimated.

A correlation between $R q$ and $D$ (and consequently with the density) could then be determined: by decreasing the surface $R q$, the NTs diameter increases and the corresponding density decreases. The lowest $R q$ achieved under $10 \mathrm{~V}$ leads to the highest and more uniform (standard deviation of $0.2 \mathrm{~nm}$ ) diameter obtained after the anodization. Accordingly, the slight $R q$ increase (for the 15 and $18 \mathrm{~V}$ samples) was also reflected in a slight decrease of the NTs diameter. The same correlation is followed by the $20 \mathrm{~V}$ sample, in which a $R q$ decrease leads to a $D$ increase.

The same parameters were also determined for the AR sample $(D=144.7 \mathrm{~nm}$ and density $=55.03$ $\mathrm{NTs} / \mu \mathrm{m}^{2}$ ). According to these relations, the NTs $D$ and density are determined by the Ti $R q$ prior to the 
anodization. However, no direct relation between $S P$ and the NTs diameter was obtained (Fig. 7). The 10 V sample has periodical ripple-like structures with a $S P$ of $97 \mathrm{~nm}$ that causes a final diameter of $157 \mathrm{~nm}$. Both doubling the ripples $S P$ (15 V sample) and decreasing $S P$ to $30 \mathrm{~nm}$ (18 V sample) lead to a $D$ decrease. However, notice that both 15 and $18 \mathrm{~V}$ samples present higher $R q$ than the $10 \mathrm{~V}$ sample, which leads us to conclude that an accurate balance between these two features $(R q$ and $S P)$ affects $D$ and the NTs organization. Such balance results in a higher and more uniform $D$ and more organized NTs distribution for $V_{\mathrm{EP}}=10 \mathrm{~V}$. As discussed before, the patterned Ti with small ripple-like structures, which act as nucleation points, forces nucleation to occur on those topographic minima. Since this periodical structure for $10 \mathrm{~V}$ has $S P=97 \mathrm{~nm}$, each valley behaves as a single nucleation site, forcing NTs nucleation to occur simultaneously in an organized manner (see Fig. 4(c); the $10 \mathrm{~V}$ sample a shows more ordered pore distribution in the initial nucleation layer) and leading to NTs with diameters of the same order ( $D$ $\sim 157 \mathrm{~nm}$ ). The NTs are then more packed and with slightly higher diameters due to an increase of the current flowing through these sites (almost no competition exists between the NTs).

Notice that increasing $S P$ (15 V sample) leads to a less optimized electric field on the resulting large valleys and thus in NTs competition during the anodization. This in turn decreases the degree of organization (NTs with no hexagonal shape) and the NTs diameter. On the other hand, by just decreasing $S P$, but increasing $R q$ (18 V sample), we obtain highly organized NTs but with much lower $D$.

A direct correlation between $R q$ and $D$ was determined, refining the usually stated linear relation between the anodization potential and $D[43,47,48]$. Using the same anodization potential of $60 \mathrm{~V}$ we can obtain different diameters just by tuning $R q$ and $S P$ using different electropolishing potentials.

\section{Conclusions}

The influence of the Ti surface topography on the anodization quality and organization was evaluated. We found that by optimizing the EP potential, the Ti surface roughness can be decrease by about $70 \%$. The combination of a lower roughness and the ripples-like structures patterned by the EP leads to the enhancement of both the degree of order and NT length. An EP under $10 \mathrm{~V}$ led to thick $\mathrm{TiO}_{2} \mathrm{NT}$ templates and to NTs bottoms with highly hexagonal array distribution.

The analysis of the anodization curves revealed the importance of the surface roughness and topography (such as SP ripple-like structures), where the valleys promote effective nucleation spots and thus the earlier emergence of NT nucleation. It was shown that Ti surfaces with lower $R q$ and adequate $S P\left(V_{E P}=10 \mathrm{~V}\right)$ lead to more homogeneous electric field over the sample surface. This causes a thinner $\delta_{b}$ that facilitates ionic electromigration, leading to an optimized steady state anodization. In fact, the use of adequate electropolishing electrochemical conditions allow a larger potential window for NT optimized formation and enhances the rate of migration of the metal/oxide interface into the substrate. Electropolishing under a potential of $10 \mathrm{~V}$ is the pre-determined parameter best suited for $60 \mathrm{~V}$ anodization since, besides decreasing $R q$ leads to the appropriated ripples spatial period. Higher EP voltages (above $10 \mathrm{~V}$ ) cause local-burning of the Ti surface originating areas with defects.

Additionally, one of the main achievements of this study is the experimental demonstration that the NTs diameter is not only associated with the anodization potential but also with the Ti surface conditions. This work revealed new overriding anodization parameters, the Ti surface roughness and $S P$ dimples topography, that can be tuned to accomplishing higher growth rate, longer NTs length and improved selforganization. It is also important to emphasize that a single anodization step was used in this method making it a straightforward application on Ti foils. 


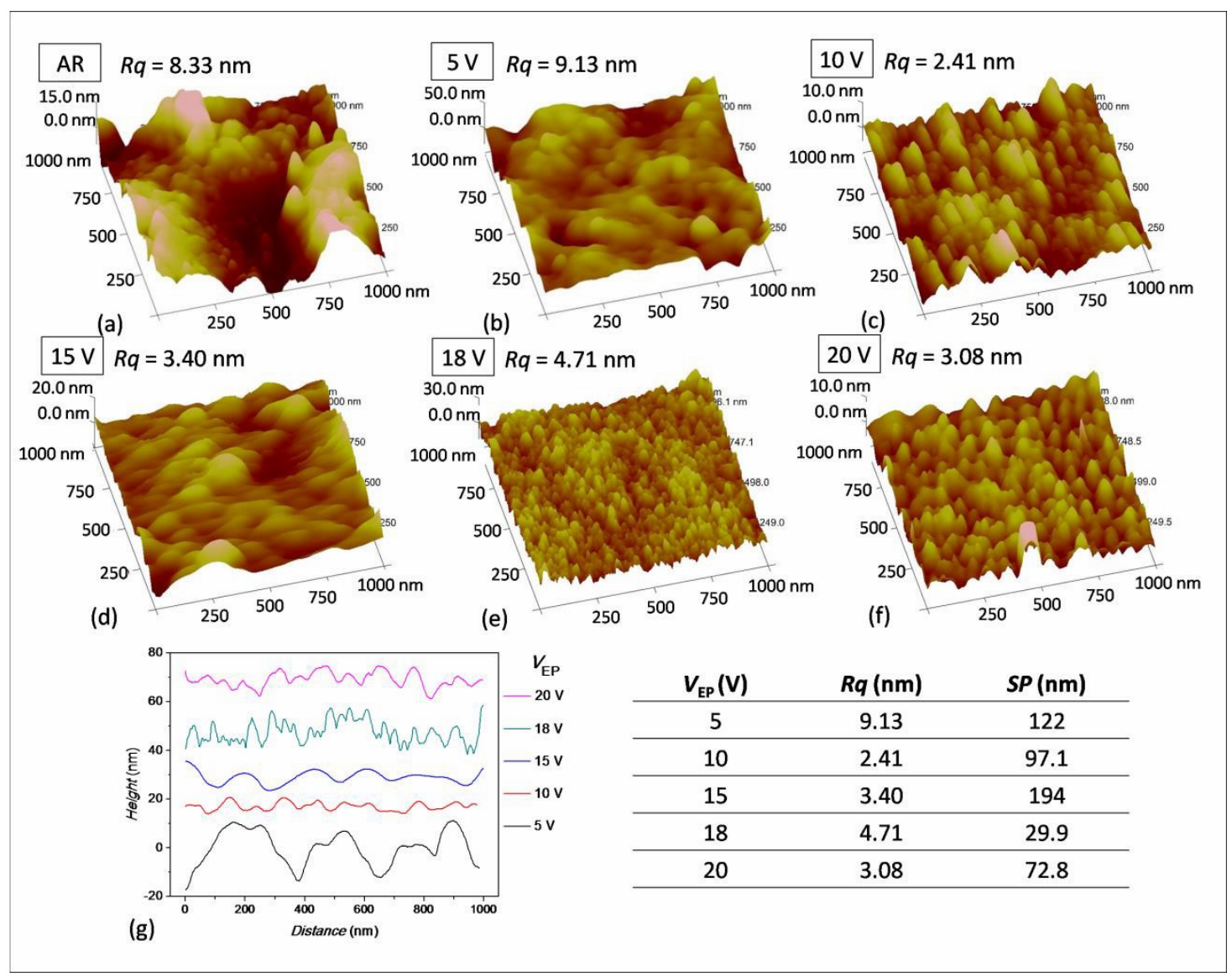

Figure 1. Ti surface topography prior to the anodization obtained using AFM and correspondent $R q$ : (a) as-rolled, (b) $5 \mathrm{~V}$, (c) $10 \mathrm{~V}$, (d) $15 \mathrm{~V}$, (e) $18 \mathrm{~V}$ and (f) $20 \mathrm{~V}$ of $V_{E P}$; (g) profile height of each sample with different $V_{E P}$; table with the Rq and SP results for each $V_{\mathrm{EP}}$ 


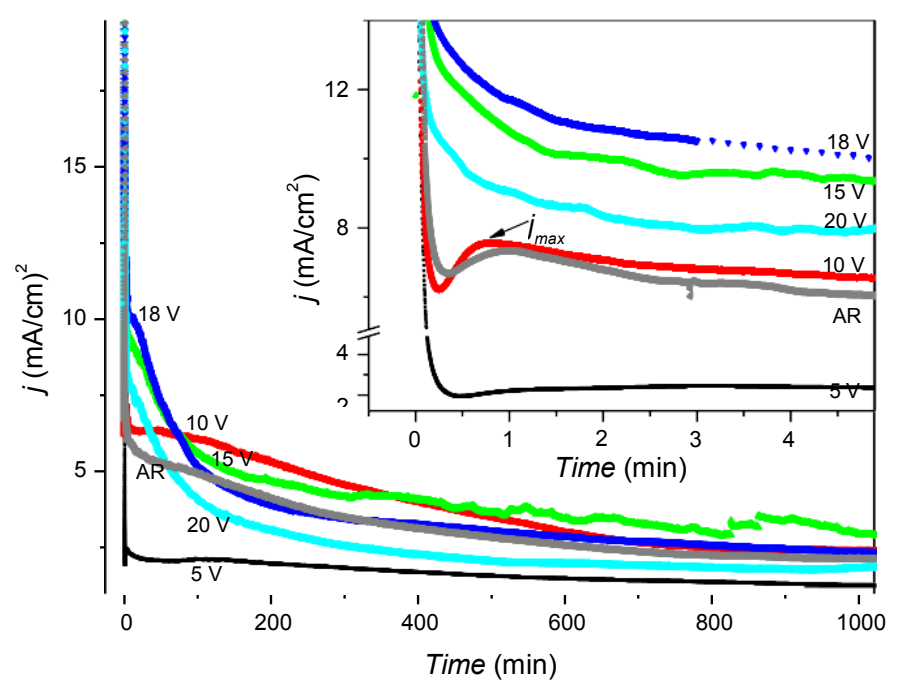

Figure 2. Current density anodization curves during the $17 \mathrm{~h}$ of anodization [inset shows the transient period (5 min)] for the AR, $5 \mathrm{~V}, 10 \mathrm{~V}, 15 \mathrm{~V}, 18 \mathrm{~V}$ and $20 \mathrm{~V}$ samples.

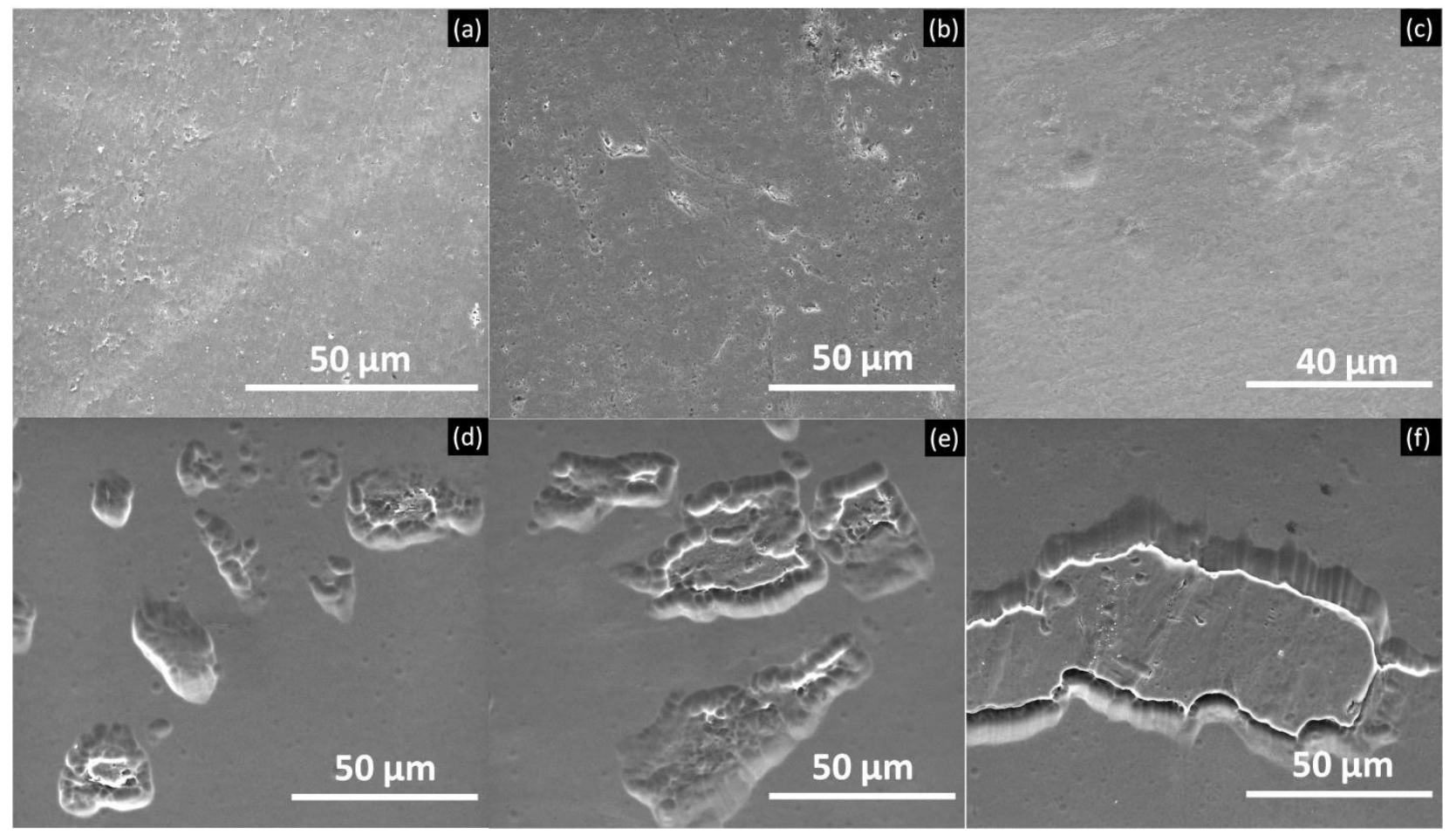

Figure 3. Top view SEM image of the Ti surfaces of the (a) AR Ti foil and electropolishing samples under (b) $5 \mathrm{~V}$, (c) $10 \mathrm{~V}$, (d) $15 \mathrm{~V}$, (e) $18 \mathrm{~V}$ and (f) $20 \mathrm{~V}$. The images of samples with $\mathrm{VEP}_{\mathrm{EP}}>10 \mathrm{~V}$ show representative areas of the whole sample surface. 


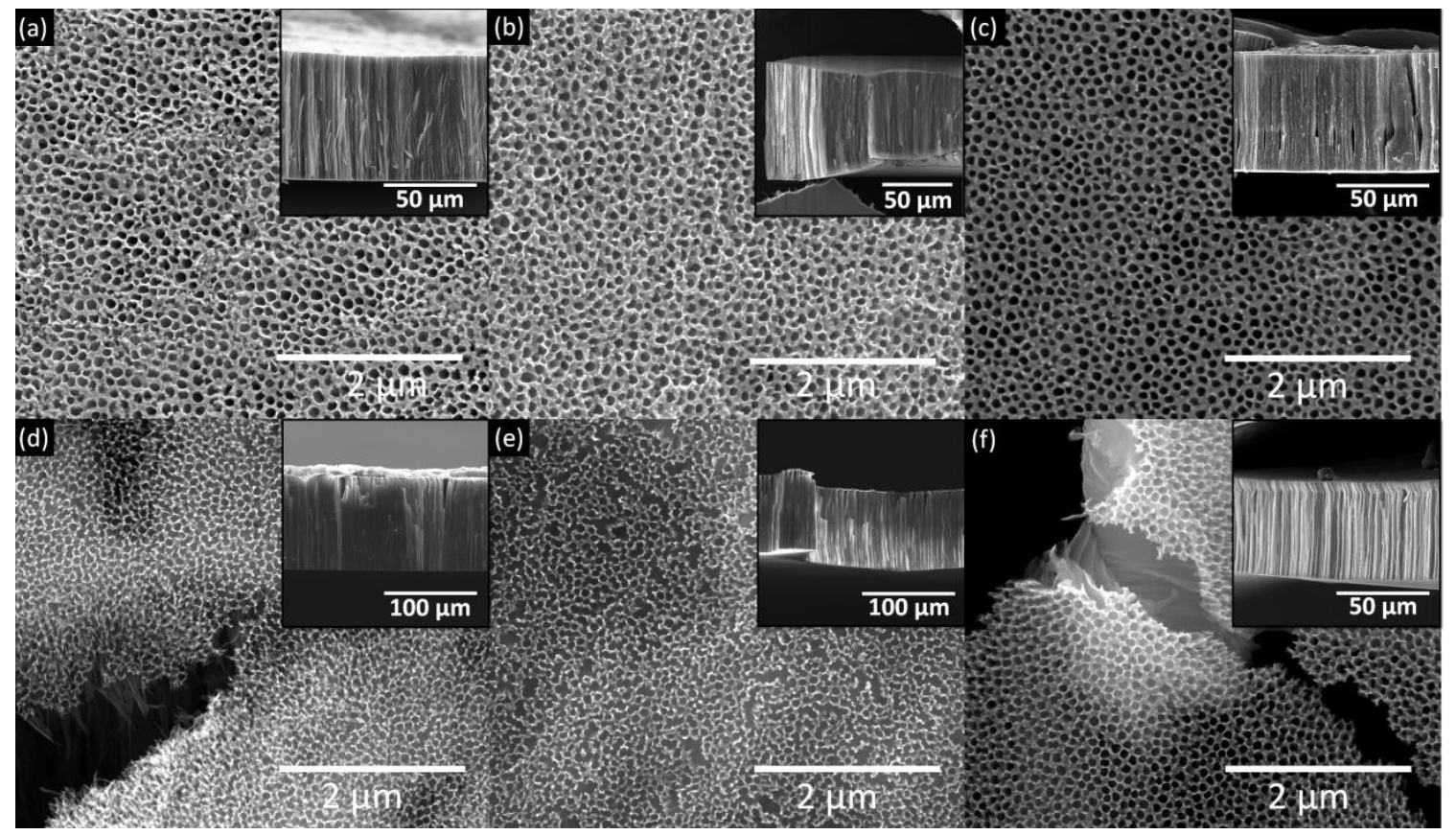

Figure 4. Top view and cross-section (insets) of the $\mathrm{TiO}_{2} \mathrm{NT}$ templates from the different samples after $17 \mathrm{~h}$ of anodization: (a) AR Ti foil, (b) $5 \mathrm{~V}$, (c) $10 \mathrm{~V}$, (d) $15 \mathrm{~V}$, (e) $18 \mathrm{~V}$ and (f) $20 \mathrm{~V}$.

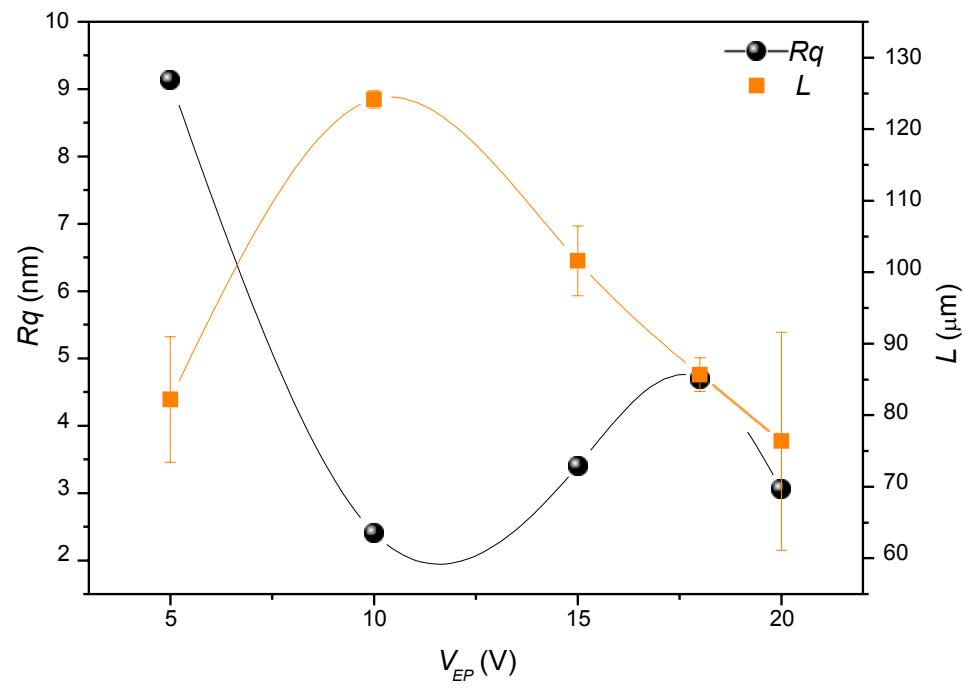

Figure 5. $\mathrm{TiO}_{2} \mathrm{NT}$ template thickness average $(L)$ and Ti surface roughness $(R q)$ as a function of $V_{E P}$. 


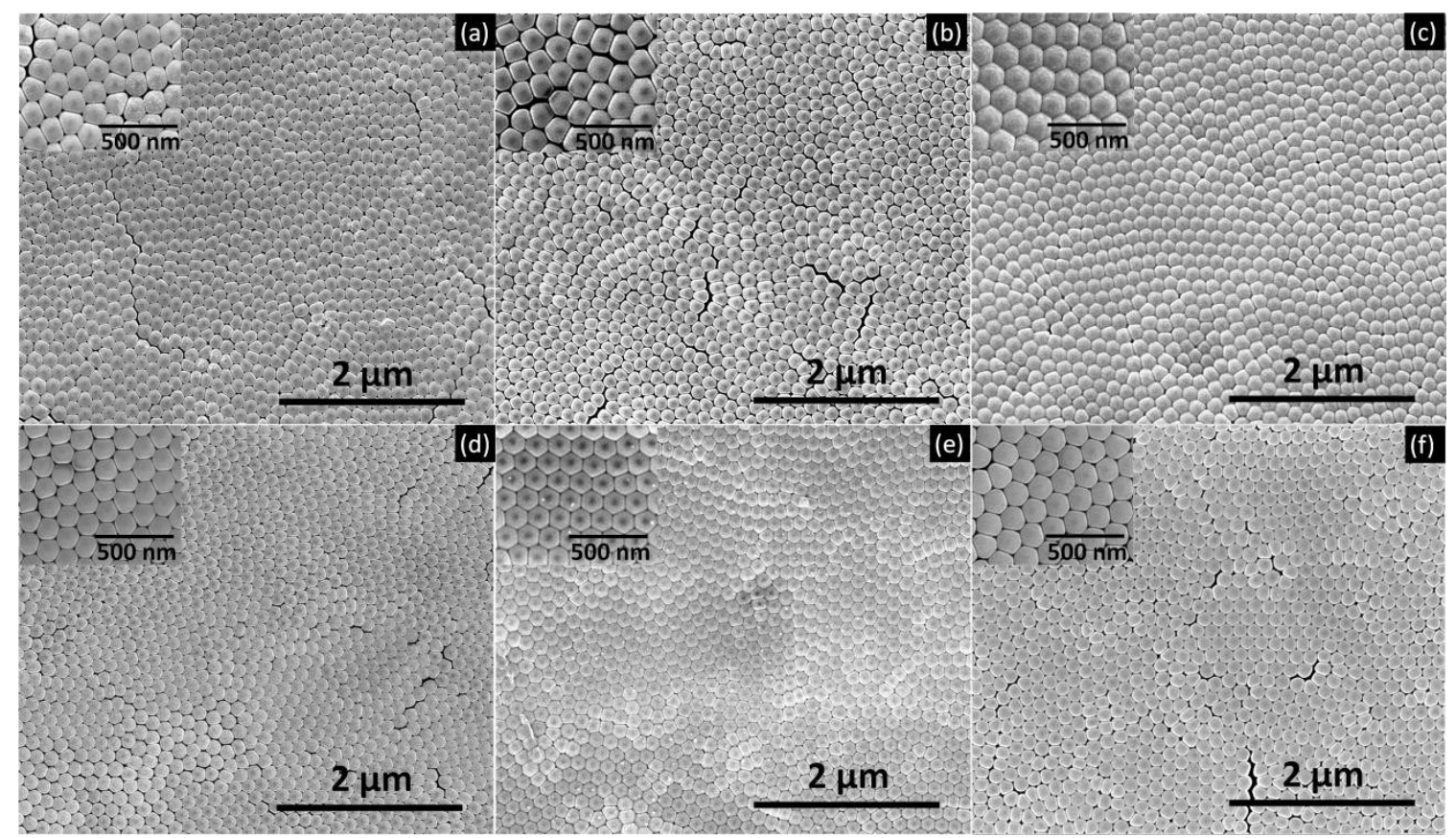

Figure 6. SEM images of the $\mathrm{TiO}_{2} \mathrm{NT}$ bottoms of the different sample: (a) AR Ti foil and after an EP of (b) $5 \mathrm{~V}$, (c) $10 \mathrm{~V}$, (d) $15 \mathrm{~V}$, (e) $18 \mathrm{~V}$ and (f) $20 \mathrm{~V}$.

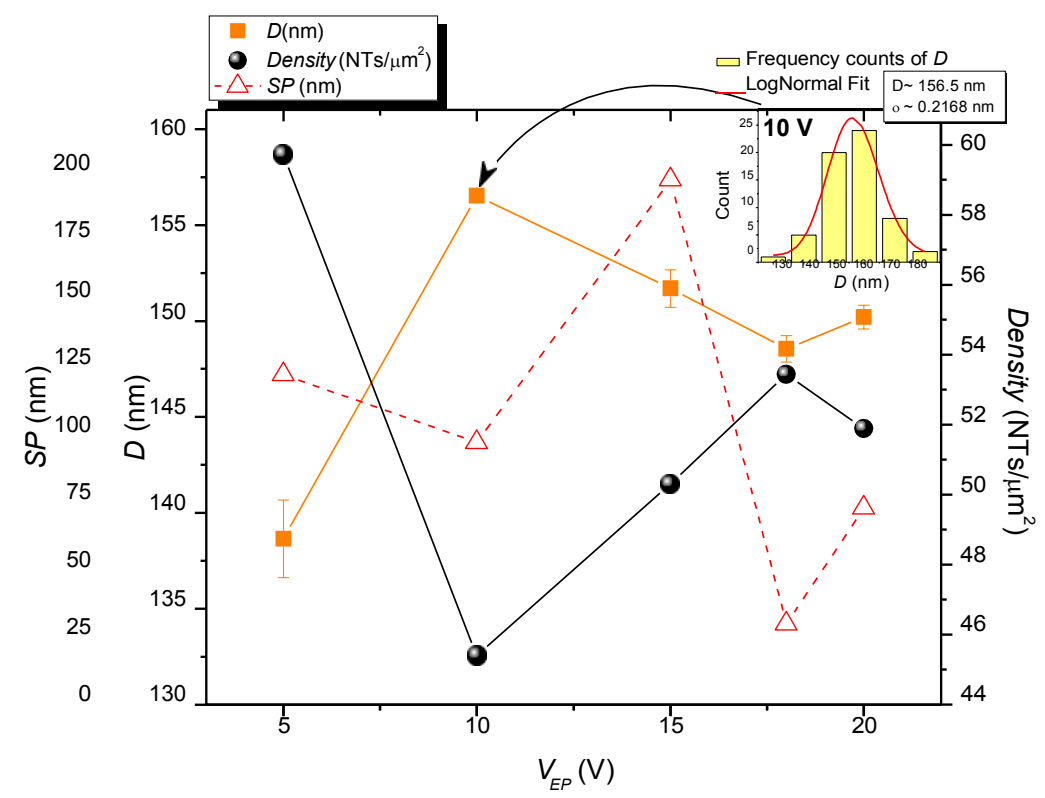

Figure7. Density [number of NTs per area $\left(\mathrm{NTs} / \mu \mathrm{m}^{2}\right)$ ], NTs diameter $(D)$, Ti electropolished ripples spacial period $(S P)$ and the $R q$ as a function of the $V_{E P} D$ and $\sigma$ (standard deviation) were calculated by performing an histogram of the NTs diameter distribution and a log-normal fit for each sample; the upper inset shows the NTs $D$ histogram and log-normal fit of the $10 \mathrm{~V}$ sample. 
Acknowledgment. A.Apolinário acknowledges the financial support under project PTDC/CTMMET/118236/2010. C. T. Sousa are also grateful to FCT for the Post-Doc fellow (reference: SFRH/BPD/82010/2011). J. Ventura acknowledges financial support through FSE/POPH. L. Andrade acknowledges European Research Council for funding within project BI-DSC - Building Integrated Dye sensitized Solar Cells (Contract number: 321315). The authors acknowledge funding from FCT through the project H2 Solar (PTDC/EQU-EQU/104217/2008) and the Associated Laboratory - IN.

\section{References}

[1] van de Krol R, Liang Y and Schoonman J 2008 J. Mater. Chem. 182311

[2] O’Regan B and Gratzel M 1991 Nature 353 737; Gratzel M 2001 Nature 414338

[3] Paulose M, Prakasam H E, Varghese O K, Peng L, Popat K C, Mor G K, Desai T A and Grimes C A 2007 J. Phys. Chem. C 111 (41) 14992

Paulose M, Shankar K, Yoriya S, Prakasam H E, Varghese O K, Mor G K, Latempa T A, Fitzgerald A and Grimes C A 2006 J. Phys. Chem. B 110 (33) 16179

[4] Shankar K, Mor G K, Prakasam H E, Yoriya S, Paulose M, Varghese O K and Grimes C A 2007 Nanotechnology 18065707

[5] Vega V, Cerdeira M A, Pride V M, Alberts D, Bordel N, Pereiro R, Mera F F, Garcia S, HernandezVelez M and Vasquez M 2008 J. Non-Cryst. Solids 3545233

[6] Balaur E, Macak J M, Taveira L and Schmuki P 2005 Electrochem. Commun. 71066

[7] Yin H, Lui H and Shen W Z 2010 Nanotechnology 21035601

[8] Sreekantan S, Saharudin K A, Lockman Z and Tzu T W 2010 Nanotechnology 21365603

[9] So S, Lee K and Schmuki P 2012 J. Am. Chem. Soc. 2811316

So S, Lee K and Schmuki P 2013 Chem. Eur. J. 192966

[10] Macak J M, Tsuchiya H and Schmuki P 2005 Angew. Chem. Int. Ed. 442100

[11] Sun L, Zhang S, Sun X W and He X J 2009 Electroanal. Chem 6637

[12] Wang D, Liu Y, Yu B, Zhou F and Liu W 2009 Chem. Mater. 211198

[13] Tang X and Li D 2009 J. Phys. Chem. C 1137107

[14] Berger S, Kunze J, Schmuki P, LeClere D, Valota A T, Skeldon P and Thompson G E 2009 Electrochim. Acta $\mathbf{5 4} 5942$

[15] Berger S, Hahn R, Roy P and Schmuki P 2010 Phys. Status Solidi b 2472424

[16] Cai Q Y, Paulose M, Varghese O K and Grimes C A 2005 J. Mater. Res. 20230

[17] Vega V, Prida V M, Hernández-Vélez M, Manova E, Aranda P, Ruiz-Hitzky E and Vázquez M

2007 Nanoscale Res. Lett. 2355

[18] Prida V M, Vega V, Aranda P, Pirota K R, Vázquez M and Ruiz-Hitzky E 2007 J. Magn. Magn. Mater. 316110

[19] Nielsch K, Choi J, Schwirn K, Wehrspohn R B and Gosele U 2002 Nano Lett. 2677

[20] Sulka G D, Kapusta-Kołodziej J, Brzozka A and Jaskuła V 2010 Electrochim. Acta 554359

[21] Macak J M, Albu S P and Schmuki P 2007 Phys. Stat. Sol. RRL 1181

[22] Shin V and Lee S 2008 Nano Lett. 83171

[23] Lee B G, Hong S Y, Yoo J E and Choi J 2011 Appl. Surf. Sci. 2577190

[24] Lu K, Tian Z and Geldmeier J A 2011 Electrochim. Acta 566014

[25] Li Y Yu X and Yang Q 2009 Journal of Sensors 2009402174

[26] Raja K S, Misra M and Paramguru K 2005 Electrochim. Acta 51154

[27] Leitao D C, Apolinario A, Sousa C T, Ventura J, Sousa J B, Vazquez M and Araujo J P $2011 \mathrm{~J}$.

Phys. Chem. C 1158567

[28] Albertin K F, Tavares A and Pereyra I 2013 Applied Surface Science 284772

[29] Landolt D 1987 Electrochim. Acta 32 1-11

[30] Rauf A, Mehmood M, Rasheed M A and Aslam M 2009 J. Solid State Electrochem. 13 321-332

[31] Landolt D, Chauvy P F, Zinger O 2003 Electrochim. Acta 483185 
[32] Konovalov V V, Zangari G and Metzger R M 1999 Chem. Mater 11 1949-1951

[33] Singh S, Barden W R T and Kruse P 2008 ACS Nano 22453

[34] Bandyopadhyay V, Miller A E, Chang H C, Banerjee G, Yuzhakov V V, Yue D F, Ricker R E, Jones S, Eastman J A, Baugher E and Chandrasekhar M 1996 Nanotechnology 7360

[35] Yuzhakov V V, Chang H C and Miller A E 1997 Phys. Rev. B 5612608

[36] Yuzhakov V V, Takhistov P V and Miller A E and Chang H C 1999 Chaos 962

[37] Asoh H, Matsuo M, Yoshihama M and Ono S 2003 Appl. Phys. Lett. 83 4408-4410

[38] Masuda H, Yamada H, Satoh M, Asoh H, Nakao M and Tamamura T 1997 Appl. Phys. Lett. 71 $2770-2772$

[39] Li A P, Muller F, Birner A, Nielsch K and Gosele U 1998 J. Appl.Phys. 84 6023-6026

[40] Kong L B, Huang Y, Guo V and Li H L 2005 Mater. Lett. 591656

[41] Apolinário A, Sousa C T, Ventura J, Costa J D, Leitão D C, Moreira J M, Sousa J B, Andrade L, Mendes A M and Araújo J P 2014 J. Mater. Chem. A 2 9067-9078

[42] Rasband W S 1997-2009 ImageJ, US National Institutes of Health, Bethesda, Maryland, USA, http://rsb.info.nih.gov/ij/

[43] Lee W, Ji R, Gosele U and Nielsch K 2006 Nature Materials 5 741-747

[44] Choi J, Wehrspohn R B, Lee J and Gösele U 2004 Electrochim. Acta 49 2645-2652

[45] Yuan X, Zheng M, Ma L and Shen W 2010 Nanotechnology 21405302

[46] Palmieri V 2003 Proceedings of the 11th Workshop on RF Supecondutivity paper WeT02

[47] Schultze J W and Lohrengel M M 2000 Electrochim. Acta 45 2499-513

[48] Macak J M, Hildebrand H, Marten-Jahns V and Schmuki P 2008 Journal of Electroanalytical

Chemistry 621 254-66 
LEPABE - Laboratory for Process Engineering, Environment, Biotechnology and Energy

Lepabe

Lalborecory fior Proceas Englineering,

\section{U.PORTO}

FEUIP FACULDADE DE ENGENHARIA DEPARTAMENTO DE UNIVERSIDADE DO PORTO $\mid$ ENGENHARIA QUIMICA 